\title{
UPMSat-2 ACDS magnetic sensors test campaign
}

\author{
Elena Rodríguez-Rojo*, Santiago Pindado, Javier Cubas, Javier Piqueras-Carreño \\ Instituto Universitario de Microgravedad “Ignacio Da Riva” (IDR/UPM), ETSI Aeronáutica y del Espacio, Universidad Politécnica de Madrid, Pza. del Cardenal Cisneros 3, Madrid \\ 28040, Spain
}

\section{A R T I C L E I N F O}

Article history:

Received 1 October 2017

Received in revised form 4 June 2018

Accepted 30 August 2018

Available online $\mathrm{xxxx}$

\section{Keywords:}

UPMSat-2 ADCS

Magnetic control

ADCS testing

ADCS integration

Space simulation

\begin{abstract}
A B S T R A C T
The present paper summarizes the testing work carried out in relation to the UPMSat-2 Attitude, Control and Determination Subsystem (ADCS) sensors. The ACDS of this satellite is based on the magnetic interaction with the Earth magnetic field, thus the selected sensors have been magnetometers. This test campaign was carried out in order to check the performance and accuracy of the magnetometers (acceptance tests) and to analyse its effect on the characteristic times of the satellite's stabilization process once its normal attitude has been perturbed (integration tests). Since the adequate calibration instruments were not available in the institute, for the acceptance tests a simplified method was designed and performed. The proposed methodology allows auto-calibrating the magnetometers, using the Earth magnetic field as reference and without a comparing with calibrated magnetometers. For the integration tests, the estimated errors were introduced in the simulator of the mission in order to analyse its effect on the stabilization time, once the satellite's attitude is perturbed. The results of the simulations indicate a correct performance of the magnetometers, as the convergence of the satellite attitude to the target one was reached within a reasonable period.
\end{abstract}

(c) 2018 Published by Elsevier Ltd.

\section{Introduction}

Although magnetic interaction has been used in attitude control for satellites since the second half of the 20th century [1], the torques obtained with this method have traditionally been restricted to being used as secondary actuators (for instance, complementing reaction wheels [2-6], or in conjunction with stabilization by gravity gradient [7-9]). More recently, some studies have gone into depth regarding the possibilities of a fully magnetic attitude control, especially for small satellites with not too demanding orientation requirements [10-13].

The magnetic control is based on the interaction between the earth magnetic field and the one produced by the satellite itself, either passively (with permanent magnets or magnetic coils [14-16]) or actively (with magnetic torquers or magnetic torque rods - hereinafter referred as magnetorquers - [17-19]). Magnetic Attitude Control Subsystems (MACSs) have some advantages over other kinds of attitude control subsystems in terms of weight and equipment needs (e.g., there is no need to carry propellant). This fact, together with the possibility of very simple designs, makes MACSs very suitable for micro, nano and pico-satellites operating in Low Earth Orbits (LEO). On the other hand, the main

\footnotetext{
* Corresponding author.

E-mail address: elena.rodriguez.rojo@upm.es (E. Rodríguez-Rojo).
}

disadvantage of MACSs lies in the limitation concerning the generated control torque, as this torque is always perpendicular to the Earth's magnetic field direction and makes it impossible to perform a continuous control in the three axes [20]. Therefore, it can be said that MACSs are always under-actuated control systems, although some studies have recently concluded that the attitude of a satellite can be controlled only with magnetic actuators in the presence of a variable magnetic field [21-24].

In addition, MACSs based on purely magnetic instruments (both sensors and actuators) are a very interesting alternative for small and low-cost satellites, due not only to their aforementioned simplicity, but also to their power efficiency and because these kind of devices does not include rotating elements (reaction wheels) and do not degrade. It should also be noted that a large number of missions have chosen this kind of control in recent times [14-17,2527].

\subsection{Overview of the UPMSat-2 mission}

The UPMSat- ${ }^{1}$ (see Table 1 and Fig. 1 ) is $50 \mathrm{~kg}$ microsatellite designed and developed at the IDR/UPM Institute of Universidad Politécnica de Madrid, for educational and technology demonstration

\footnotetext{
1 The UPMSat- 2 was preceded by a former one, the UPMSat-1, developed by the same team and launched in 1995.
} 
Table 1

General characteristics of the UPMSat-2 mission [28].

\begin{tabular}{|c|c|}
\hline Mission Life & 2-year \\
\hline Orbit & $\begin{array}{l}\text { Sun-synchronous: } \\
\text { - Noon. } \\
\text { - Altitude: } 600 \mathrm{~km} \\
\text { - Period: } 5828 \mathrm{~s}\end{array}$ \\
\hline Mass & $50 \mathrm{~kg}$ \\
\hline Dimensions & $0.5 \mathrm{~m} \times 0.5 \mathrm{~m} \times 0.6 \mathrm{~m}$ \\
\hline Attitude Control & $\begin{array}{l}\text { Magnetic: } \\
\text { - SSBV magnetometers } \\
\text { - ZARM Technik AG magnetorquers } \\
\text { - Control law designed by IDR/UPM. }\end{array}$ \\
\hline Thermal Control & Passive \\
\hline Power & $\begin{array}{l}\text { Based on solar photovoltaic panels and batteries: } \\
\text { - } 5 \text { body-mounted solar panels (Selex Galileo SPVS- } \\
5 \text { modules with Azur Space 3G28C triple junction } \\
\text { solar cells) } \\
\text { - Li-ion battery designed by SAFT } \\
\text { - Direct Energy Transfer (DET) }\end{array}$ \\
\hline $\begin{array}{l}\text { On board Electronic } \\
\text { Box (E-BOX) }\end{array}$ & $\begin{array}{l}\text { Based on FPGA (designed by Tecnobit S.L. and } \\
\text { programmed by STRAST/UPM). Includes: } \\
\text { - On-board computer } \\
\text { - Data handling } \\
\text { - Power supply control } \\
\text { - Power supply distribution }\end{array}$ \\
\hline Communications & $\begin{array}{l}\text { - Link at } 436 \text { Mhz frequency } \\
\text { - } 4 \text { monopole antennae system } \\
\text { - Emsys communication card installed in the E- } \\
\text { BOX } \\
\text { - Ground station software programmed by STRAST, } \\
\text { hardware configuration supervised by INTA }\end{array}$ \\
\hline Payloads & $\begin{array}{l}\text { - Bartington magnetometer } \\
\text { - SSBV rotation wheel } \\
\text { - Iberespacio thermal microswitch } \\
\text { - Solar sensors } \\
\text { - Tecnobit on-board computer (E-BOX) }\end{array}$ \\
\hline
\end{tabular}

purposes. The launch is expected by the end of 2018 or beginning of 2019. More information on this mission can be found on [28-30].

A purely magnetic Attitude, Control and Determination Subsystem (ACDS) has been selected for the UPMSat-2, as it is both simple and reliable. The design of this subsystem includes three ZARM (ZARM Technik AG) magnetorquers, and two SSBV (SSBV Space \&
Ground Systems) fluxgate magnetometers. The second magnetometer is carried on board to increase the reliability of the subsystem. Furthermore, the information from a third fluxgate magnetometer by Bartington, present on board as payload, is available for the ADCS during the mission (see Fig. 2). The programmed control law is based on a modification of the "B-dot" strategy, with this modification allowing the satellite angular rotation rate to be controlled, and also the setting of the rotation axis of the satellite perpendicular to the satellite orbit's plane (see [31] for more information). The satellite is planned to orbit in a sun-synchronous noon/midnight orbit in which the sun radiation will be almost perpendicular to the aforementioned rotation axis ( $Z$ axis of the UPMSat-2), thereby allowing a better thermal control and a high orbit-average energy production (solar panels are located at $+X$, $-X,+Y$ and $-Y$ axes of the UPMSat-2, see Fig. 1).

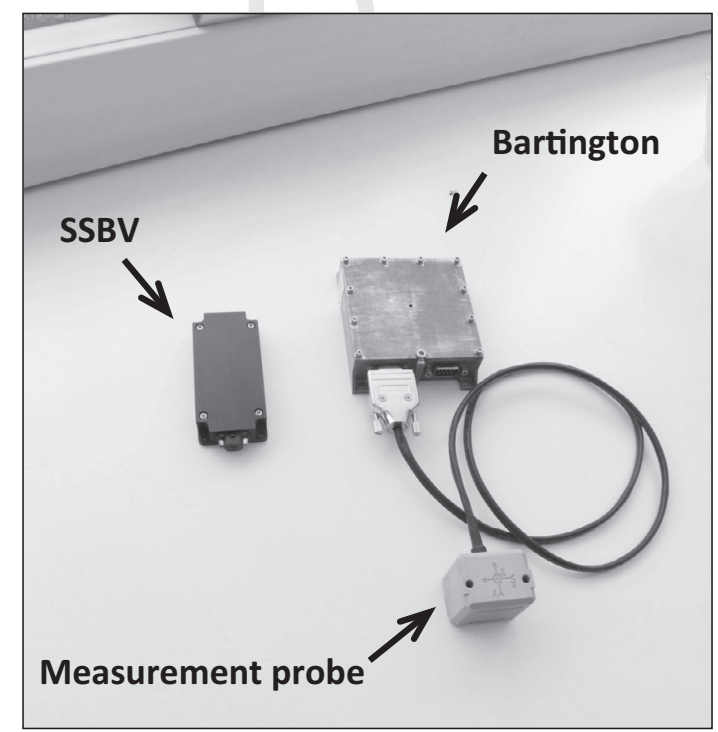

Fig. 2. Magnetometers of the UPMSat-2 satellite ADCS. One of the mission SSBV magnetometer (on the left side), and the payload Bartington magnetometer with its measurement probe attached (on the right side).

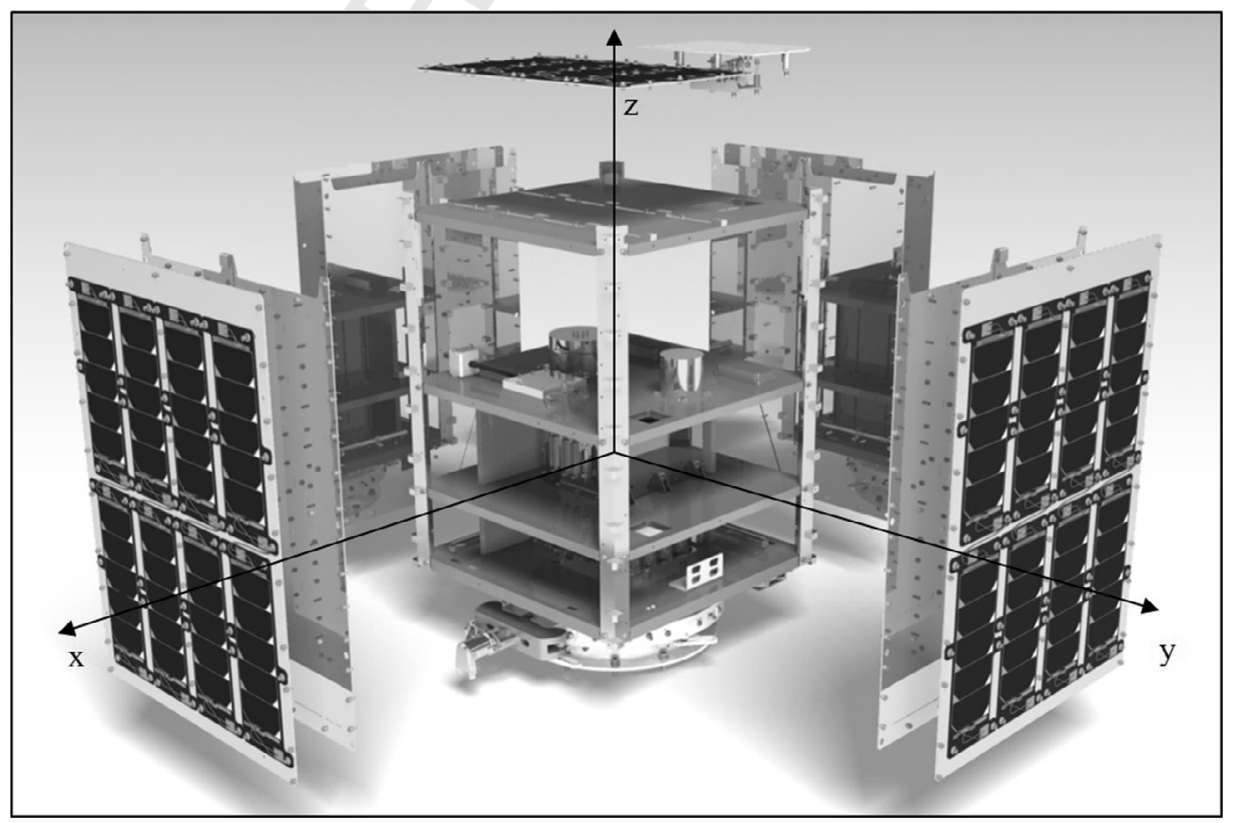

Fig. 1. CAD drawing of the UPMSat-2 satellite. 


\subsection{Aim of the present work}

In this work, the measurement process carried out to verify the correct performance of the UPMSat-2 magnetometers is described, in addition to the analysis of their effect in characteristic times of the satellite's stabilization process. This process has been applied as part of the acceptance and integration tests plan of the UPMSat-2 ADCS. It should be underlined that the acceptance test plan should be performed on every unit received from an external supplier in a space mission [32].

Once the verification was carried out, the effect of the magnetometers errors measured on the UPMSat- 2 performances was studied by Monte Carlo analyses carried out with the ADCS simulator of this satellite. It should be pointed out that this technique has been successfully used by many other authors to validate different satellite control subsystems [3,33-46].

As previously mentioned, professional procedures followed to perform a magnetometer calibration require a high-tech and dedicated facility with high-level accuracy instrumentation. The calibration methods generally rely on non-magnetic levelled turntables and Helmholtz coils. See the works by Jankowski \& Sucksdorff [47], Loubser \& Newitt [48], and Bowditch [49] for more information. Another way of addressing the magnetometer acceptance problem would have been to use a reference magnetometer. Taking into account the UPMSat-2 academic project scope, we decided to keep magnetometer acceptance tests as simple as possible. Therefore, the use of a reference magnetometer was discarded.

The tests performed in the present work verify the proper and correct UPMSat-2 magnetometers' performance behaviour, based on the calibration provided by the manufacturer. These tests provide a quite accurate estimation of the sensors error level, in the aim of verifying the UPMSat- 2 ADCS robustness. The magnetometer calibration acceptance depends on the ADCS ability to filter the possible errors in the sensor measurements. An alternative magnetometers calibration, once integrated into the satellite, is now being developed by the authors at the IDR/UPM Institute.

Finally, one of the goals of the present work is to demonstrate that control subsystem robustness acceptance procedure can be undertaken with limited means. Furthermore, it should be highlighted that the research carried out within this work will be included in the academic program of the Master in Space Systems at Universidad Politécnica de Madrid, in agreement with the continuous improvement requirements stated at the Master's quality assurance program.

The present work is organised as follows: the testing procedure is defined in Section 2, whereas the results are included and discussed in Section 3. In Section 4 the aforementioned Monte Carlo analysis to study the effect of these errors on the UPMSat-2 control subsystem performance is included. Finally, conclusions are summarized in Section 5.

\section{Testing procedure description}

\subsection{The Earth's magnetic field}

In order to check the performance of a magnetic sensor, the magnetic field that interacts with it needs to be well characterized. In the present work, the Earth magnetic field is used as reference to analyse the magnetometers' performance (see Fig. 3). As a high accuracy magnetometer was not available to characterize the aforementioned Earth magnetic field, a well-established analytical model was used to obtain the local values of this magnetic field instead.

There are analytical models of the Earth's magnetic field which take into account the Main Field (Earth magnetic field is modelled

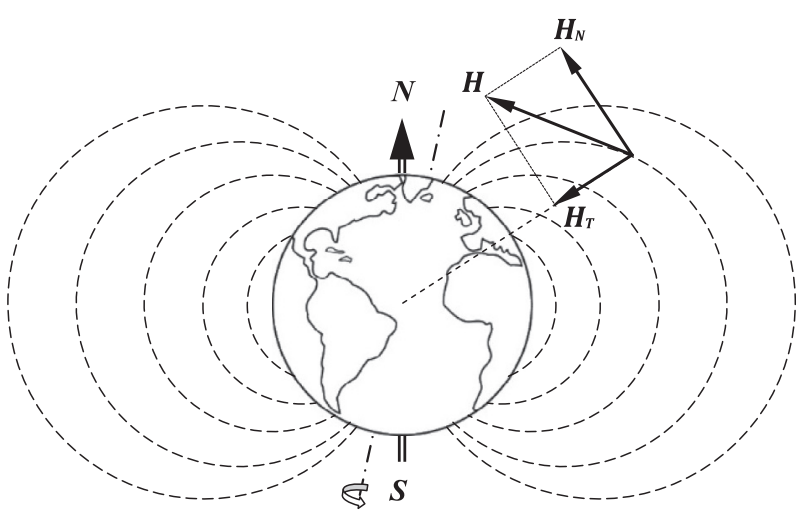

Fig. 3. Sketch of the Earth geomagnetic field lines. The local field vector, $\boldsymbol{H}$, is broken down into two components, one towards the centre of the Earth, $\boldsymbol{H}_{T}$, and the other one parallel to its surface, $\boldsymbol{H}_{\boldsymbol{N}}$.

as the field created by a magnet centred in the centre of the Earth, $90 \%$ of the total Earth magnetic field), as well as other contributions (solar flux in high altitudes, or the contribution of the Earth crust), including the evolution over time [50]. The most wellknown models are the World Magnetic Model, WMM, and the Geomagnetic Reference Field, IGRF. The main difference between the two models is that the WMM model is a predictive model and the IGRF model is an historic model. These models are valid for periods of five years. For practical reasons we selected the IGRS model of the magnetic scalar potential, $\psi$, for this work:

$\psi(r, \theta, \lambda, t)=R \sum_{l=1}^{L} \sum_{m=0}^{l}\left(\frac{R}{r}\right)^{l+1}\left[g_{l}^{m} \cos (m \lambda)+h_{l}^{m} \sin (m \lambda)\right] P_{l}^{m}(\cos \theta)$,

where $L$ is the maximum degree of the expansion, $\lambda$ is the East longitude, $\theta$ is the colatitude, $R$ is the Earth's radius, $g_{l}^{m}$ and $h_{l}^{m}$ are Gauss coefficients, which are functions of time, and $P_{l}^{m}$ are Schmidt normalized associated Legendre functions, of degree $l$ and order $m$. Once the magnetic scalar potential is defined, the geomagnetic field $\boldsymbol{H}$ can be easily derived:

$H=-\nabla \psi$

The errors of the modelled Earth magnetic field were considered in the present work, the typical values being $\pm 30^{\prime}$ of arc for the alignment and $\pm 200 \mathrm{nT}$ for the field intensity.

\subsection{Magnetometer performance modelling}

The magnetometers considered in the present study are three axes fluxgate magnetometers that measure the magnetic field vector, $\boldsymbol{B}_{m}$, with its components expressed in the sensor body axis, in Volts. However, these vector components do not directly represent the measured geomagnetic field, $\boldsymbol{H}_{\boldsymbol{m}}$, (expressed in $\mathrm{nT}$ ), the following transfer function being required:

$H_{m}=C^{-1} \cdot\left(B_{m}-b\right)$

where $\boldsymbol{C}^{-1}$ is a symmetric matrix (expressed in $\mathrm{nT} \cdot \mathrm{tV}^{-1}$ ), and $\boldsymbol{b}$ is the offset vector (expressed in V). The above equation is defined by means of a calibration process.

The errors that can corrupt the magnetometer measurements include biases, scale factors and misalignments. They can be classified into two main groups: environmental and instrumental errors, as seen in $[35,36]$.

Regarding the environmental perturbations, they are divided into hard iron and soft iron errors. The hard iron errors are produced by permanent magnets, magnetic hysteresis and slowly 
time-varying fields, and they can be modelled as a deviation of the value of the magnetic field, that is, a constant bias, $\boldsymbol{b}^{\boldsymbol{h} \boldsymbol{i}}$. Besides, the soft iron errors are caused by the interaction that ferromagnetic materials with external magnetic fields present. The inner field created by the ferromagnetic materials results in a change of intensity and direction of the magnetic field vector, that can be expressed with the rotation matrix, that we will call $C^{s i}$.

$b^{h i}=\left[\begin{array}{lll}b_{x}^{h i} & b_{y}^{h i} & b_{z}^{h i}\end{array}\right]$

$C^{s i}=\left[\begin{array}{lll}c_{11} & c_{12} & c_{13} \\ c_{12} & c_{22} & c_{23} \\ c_{13} & c_{23} & c_{33}\end{array}\right]$

Introducing the instrumental errors, they are composed of a bias, a scale factor and a misalignment error. The bias error is produced by fabrication deficiencies that are taken into account as a constant bias $b^{s o}$. The scale factor shows the uncertainty in the knowledge of the constant of proportionality that relates the sensor input and output, and it is represented by a diagonal matrix $\boldsymbol{S}$. The misalignment error is caused by the inability to have three perfectly perpendicular sensor axes, and it is taken into account with the matrix $\boldsymbol{E}$. The expressions of the mentioned vector and matrices are:

$b^{s o}=\left[\begin{array}{lll}b_{x}^{h i} & b_{y}^{h i} & b_{z}^{h i}\end{array}\right]$

$S=\left[\begin{array}{ccc}S_{x} & 0 & 0 \\ 0 & S_{y} & 0 \\ 0 & 0 & S_{z}\end{array}\right]$

$E=\left[\begin{array}{ccc}1 & \varepsilon_{x y} & \varepsilon_{x z} \\ \varepsilon_{x y} & 1 & \varepsilon_{y z} \\ \varepsilon_{x z} & \varepsilon_{y z} & 1\end{array}\right]$

Considering all the aforementioned errors, the full measurement model can be expressed as:

$B_{m}=S \cdot E \cdot\left(C^{s i} \cdot H_{m}+b^{h i}\right)+b^{s o}+v$

where $\boldsymbol{v}$ stands for a noise vector. The terms of the above expression can be grouped in order to reach a simpler equation, similar to the ideal Eq. (3):

$B_{m}=C^{*} \cdot H_{m}+b^{*}$

The purpose of the UPMSat-2 magnetometers is to provide the value of the measured geomagnetic field, $\boldsymbol{H}$, as a function of the output voltage, $B_{m}$. Thus, the Eq. (10) is rewritten as:

$H_{m}=C^{*-1} \cdot\left(B_{m}-b^{*}\right)$

where the noise contribution is taken into account by the first and second terms of the right side of the above equation. Commonly, $C^{*-1}$ is called the calibration matrix, whereas, as said, $\boldsymbol{b}^{*}$ is the offset vector. The manufacturer normally provides the instrumental errors, either in the form of a calibration matrix and an offset vector or in the form of the different error matrixes (scale, misalignment) and the offset. Therefore, in the acceptance test, in order to verify the by-default calibration, the environmental errors should be minimized.

\subsection{Testing procedure}

The testing procedure of magnetometers is normally carried out as described in the aforementioned references [47,48] or [49]. In the present work, the testing procedure was similar and consisted of placing the magnetometer (or its measurement probe in the case of testing the Bartington magnetometer) on a horizontal plane, with one of its axis vertically aligned and pointing towards the Earth, and the other one pointing towards the magnetic North (see Fig. 4). Each set of measurements was carried out rotating the magnetometer in relation to the vertical axis and taking measurements in steps of $\Delta \alpha=30^{\circ}$. The total number of cases tested is summarized in Table 2.

As mentioned above, the supplier company of the magnetometer sensors provides the instrumental errors, such that the true values of the magnetic field can be obtained in the absence on environmental errors. Hence, the acceptance test of the sensor should be carried out limiting as much as possible the effect of the sources of environmental errors. These possible sources, as mentioned in previous subsections, are magnets, ferromagnetic materials and time-depending magnetic fields, which can be found mostly in electronic devices and ferromagnetic materials, as tools, screws, bolts, power lines, sewage systems, etc. Therefore, it seems reasonable to state that testing inside buildings should be avoided. In the present testing campaign measurements were carried out inside a laboratory, as well as outdoors, in order to analyse the errors associated to indoor testing.

In Fig. 5, the theoretical calculation of the projection of the Earth magnetic field $\boldsymbol{H}$ (the modulus value is provided by NOAA organization) on each one of the magnetometer axes is shown, together with the vector modulus, as a function of the magnetometer rotation angle in relation to the magnetic North, $\alpha$.

Once a test case is carried out, the voltage measurements from the magnetometers have been transformed into magnetic field measurements using the transfer function (Eq. (11)) provided by the magnetometer's manufacturer. The obtained values are compared with the theoretical values explained above. In the perfect scenario, the measured values of the components of the magnetic field should match the ones from Fig. 5, the theoretical values of the components of the magnetic field.

The outdoor experimental set up is shown in Fig. 6. The magnetometer to be tested was allocated on a horizontal non-

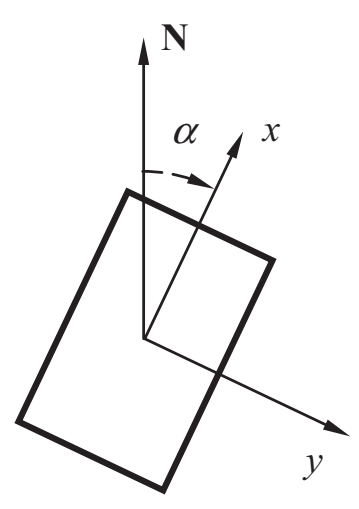

Please cite this article in press as: E. Rodríguez-Rojo et al., UPMSat-2 ACDS magnetic sensors test campaign, Measurement (2018), https://doi.org/10.1016/ j.measurement.2018.08.069

Fig. 4. Sketch of the magnetometer (or its measurement probe, in the case of the Bartington magnetometer -see Fig. 2-) placed for being tested on a horizontal surface. Initial position $\left(\alpha=0^{\circ}\right): x$-axis pointing North, $y$-axis pointing East. 
Table 2

Testing cases analysed in the campaign carried out to study SSBV and Bartington magnetometers.

\begin{tabular}{|c|c|c|c|}
\hline Unit & Case & Indoor/Outdoor & Description \\
\hline SSBV-FM008 & $\begin{array}{l}\text { FM008-1 } \\
\text { FM008-2 } \\
\text { FM008-3 } \\
\text { FM008-4 } \\
\text { FM008-5 }\end{array}$ & $\begin{array}{l}\text { Indoor } \\
\text { Indoor } \\
\text { Indoor } \\
\text { Outdoor } \\
\text { Outdoor }\end{array}$ & $\begin{array}{l}\text { Initial position: } x \text {-axis pointing West, } z \text {-axis pointing South. Clockwise rotation } \\
\text { Initial position: } x \text {-axis pointing West, } y \text {-axis pointing North. Clockwise rotation } \\
\text { Initial position: } y \text {-axis pointing North, } z \text {-axis pointing West. Clockwise rotation } \\
\text { Initial position: } x \text {-axis pointing West, } y \text {-axis pointing North. Clockwise rotation } \\
\text { Initial position: } y \text {-axis pointing North, } z \text {-axis pointing West. Clockwise rotation }\end{array}$ \\
\hline Bartington & $\begin{array}{l}\text { Bart-1 } \\
\text { Bart-2 } \\
\text { Bart-3 } \\
\text { Bart-4 } \\
\text { Bart-5 } \\
\text { Bart-6 }\end{array}$ & $\begin{array}{l}\text { Indoor } \\
\text { Indoor } \\
\text { Indoor } \\
\text { Outdoor } \\
\text { Outdoor } \\
\text { Outdoor }\end{array}$ & $\begin{array}{l}\text { Initial position: } x \text {-axis pointing North, } y \text {-axis pointing East. Clockwise rotation } \\
\text { Initial position: } y \text {-axis pointing East, } z \text {-axis pointing South. Clockwise rotation } \\
\text { Initial position: } x \text {-axis pointing East, } z \text {-axis pointing South. Clockwise rotation } \\
\text { Initial position: } x \text {-axis pointing North, } y \text {-axis pointing East. Clockwise rotation } \\
\text { Initial position: } y \text {-axis pointing West, } z \text {-axis pointing North. Clockwise rotation } \\
\text { Initial position: } x \text {-axis pointing West, } z \text {-axis pointing North. Clockwise rotation }\end{array}$ \\
\hline SSBV-FM007 & FM007-1 & Outdoor & Initial position: $x$-axis pointing West, $y$-axis pointing North. Clockwise rotation \\
\hline
\end{tabular}

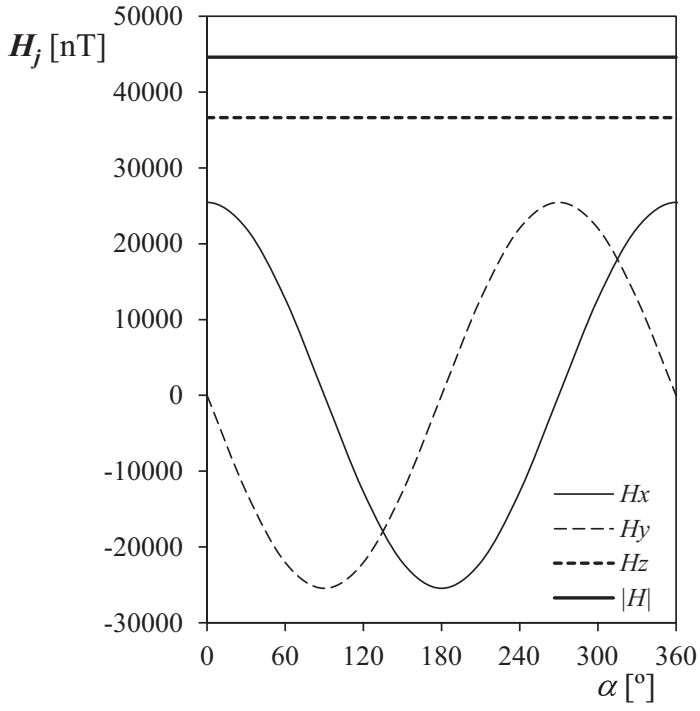

Fig. 5. Projection of the geomagnetic field, $\boldsymbol{H}$, in Madrid (Spain), on a magnetometer (or its measurement probe) body axes with the attitude sketched on Fig. 4 (i.e., a vertical rotation around $Z$ body axis). ferromagnetic turntable, which was attached to a photography tripod. In order to detach the magnetometer from the iron parts of the tripod (and reduce this possible interference), a $15 \mathrm{~cm}$ high non-ferromagnetic interface was constructed between the horizontal turntable and the aforementioned tripod. Several tests were performed, so as to determine a minimum distance of separation between the magnetometer and the rest of the test equipment (the computer and the ferromagnetic tripod head mainly), so as to minimize the interference of the test equipment in the magnetometer, $10 \mathrm{~cm}$ turned out to be this minimum distance, with a safety margin of $5 \mathrm{~cm}$, thus $15 \mathrm{~cm}$ was the distance selected.

The magnetometers were connected to a DC power supply in the tests carried out indoor, whereas lithium-ion-polymer batteries were the power supply in the case of the outdoor tests. The voltage measurements were carried out by using a National Instruments acquisition system connected to a computer. In each measurement 1000 samples were taken at $100 \mathrm{~Hz}$ frequency. The process was controlled with software programmed in LABVIEW.

\section{Results}

In Fig. 7 the results from two of the tests carried out on both, one of the SSBV magnetometers (FM008) and the Bartington magnetometer, are shown:

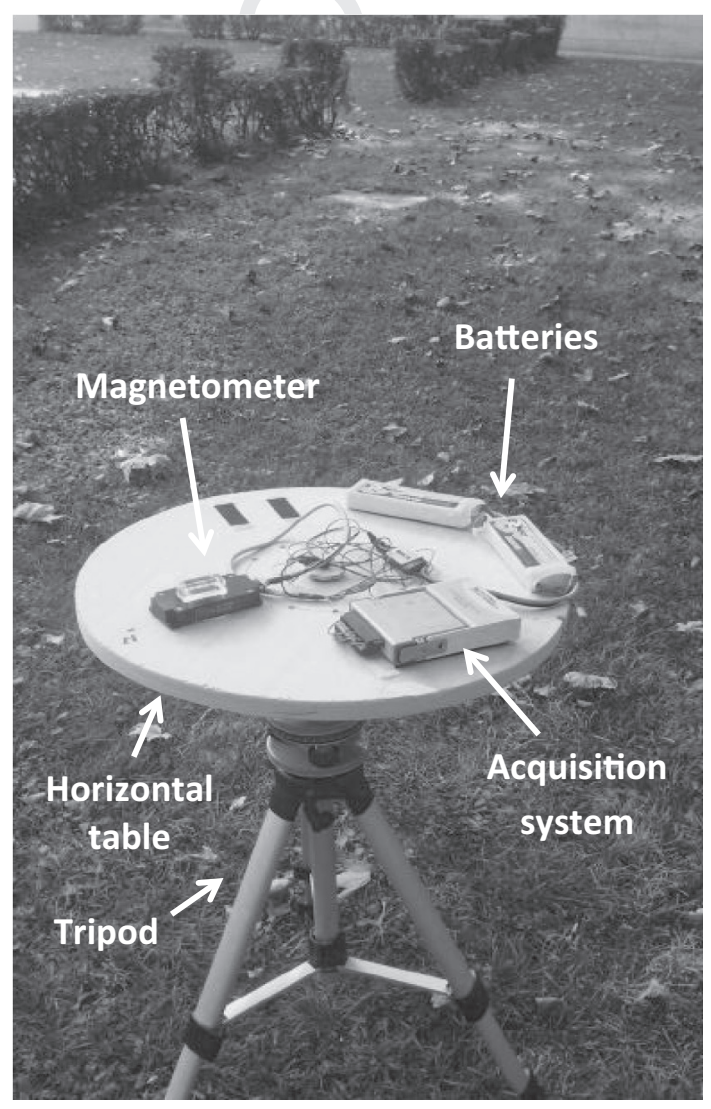

Fig. 6. Outdoor experimental setup prepared to carry out a set of measurements.

- Case FM008-2 (indoor).

- Case FM008-4 (outdoor).

- Case Bart-1 (indoor).

- Case Bart-4 (outdoor).

A better accuracy can be appreciated from the outdoor testing in the graphs included in this figure, with this differences being especially significant regarding the vertical axis. Presumably, this better results obtained in the outdoor testing when compared to the indoor testing, are the result of the reduction on the magnetic disturbance caused by the iron structure of the building where the indoor testing was performed.

In order to estimate the level of the aforementioned lack of accuracy, the angle $\gamma$ between the geomagnetic field, $\boldsymbol{H}$, and the measured values, $\boldsymbol{H}_{\boldsymbol{m}}$, and the percentage difference between the modulus of both vectors: 

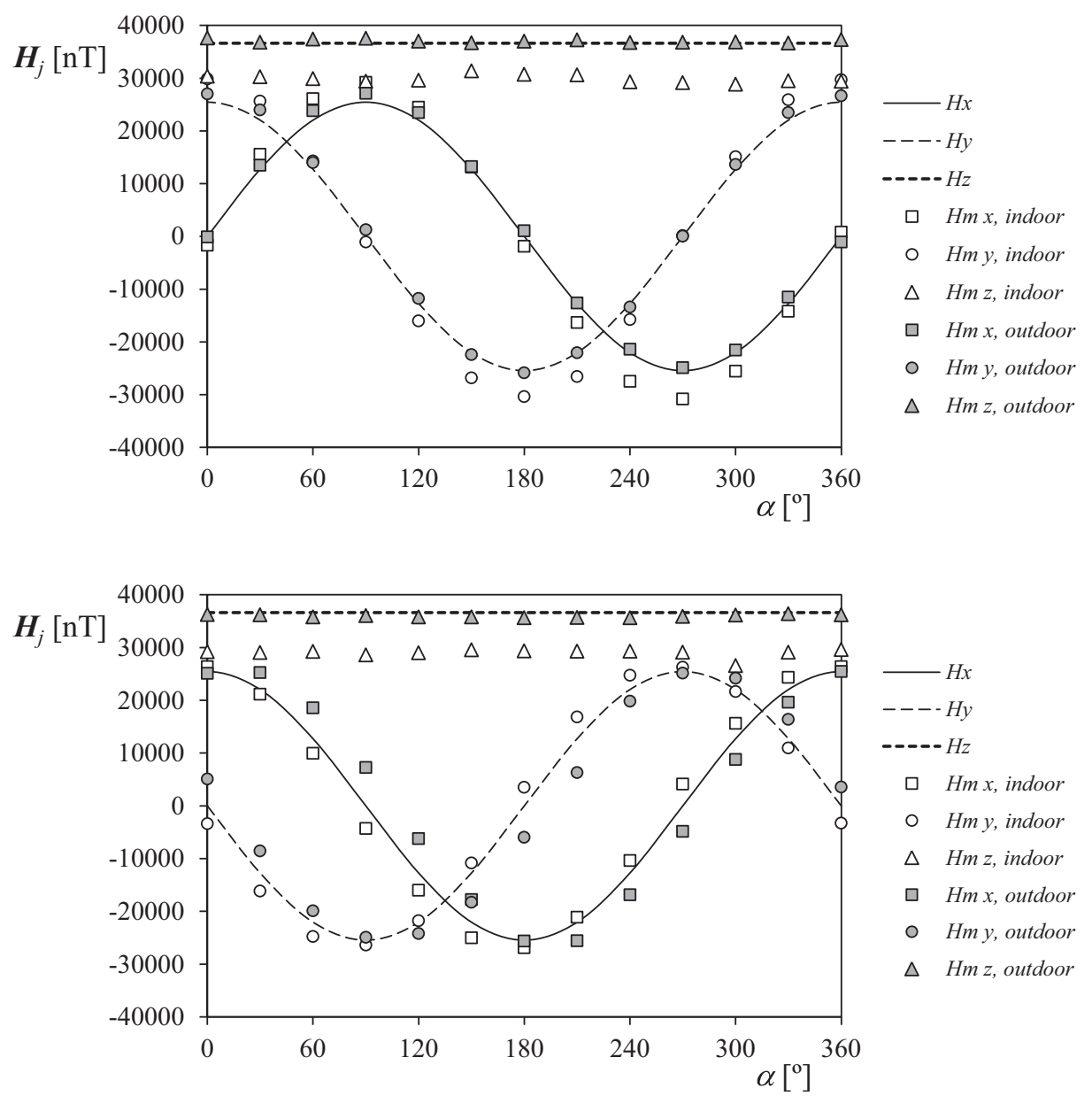

Fig. 7. Components of the geomagnetic field $\boldsymbol{H}_{\boldsymbol{m}}$ measured indoor and outdoor, in relation to the angular position of the magnetometer (or its measurement probe), (see Fig. 4 and Table 2). The measured values are compared to the corresponding ones from the IGRS model, $\boldsymbol{H}$ (depicted in solid and dashed lines). Top: SSBV magnetometer (cases FM008-2 and FM008-04). Bottom: Bartington magnetometer (cases Bart-1 and Bart-4).

$\Delta|H|=\frac{\left|H_{m}\right|-|H|}{|H|}$

were calculated. In Fig. 8 the results are shown. Based on this figure, it can be said that the pointing errors can be estimated in a first approximation as being between $\gamma=2^{\circ}$ and $\gamma=10^{\circ}$, whereas the magnetic field, can be measured with an error within the brackets from $\Delta|\boldsymbol{H}|=-15 \%$ to $\Delta|\boldsymbol{H}|=5 \%$ (approximated values). These values include cases both indoor and outdoor.

If we only take into consideration the outdoor cases, the deviations values, $\gamma$ and $\Delta|\boldsymbol{H}|$, are smaller: $\Delta|\boldsymbol{H}|$ varies between $-5 \%$ and $5 \%$ approximately, and $\gamma$ takes values between $1^{\circ}$ and $4^{\circ}$ approximately (we have not taken into account Bart-4 outdoor case in these calculations because it presents a behaviour significantly different from the other outdoor cases; probably the test scenario was not appropriate in this case).

It can be deduced that the interferences that the magnetometer suffers in the indoor cases affect the measurements of the instrument. Thus, for magnetometer characterizations, the test should be undertaken outdoor and as far as possible from ferromagnetic interferences (buildings, sewage system, street lamps...).

\section{Effect of the magnetometers error on the UPMSat-2 performance}

The effect of the magnetometers errors detected during the testing campaign on the UPMSat- 2 performances was analysed using Monte Carlo techniques, in order to establish if the ACDS and especially its control law are robust enough to correct any perturbation on the foreseen satellite's attitude (see Fig. 9).

\subsection{Magnetometer errors definition}

The results of the tests performed to the SSVB and Bartington magnetometers have been used to determine a baseline of errors introduced by these sensors in the ADCS. The tests corresponding to FM008-4 and FM008-5 cases, i.e., two of the tests performed outdoors on one of the nominal magnetometers, FM008, were selected as representative cases. From the data three different errors were defined:

(1) Misalignment error, . Defined as the differences between the direction of the geomagnetic field, $\boldsymbol{H}$, and the one measured by the magnetometer. The maximum misalignment error considered in the simulations is $\gamma=2^{\circ}$. The misalignment error is related to misalignment error from Eq. (8) and soft iron environmental perturbations from Eq. (5).

(2) Offset error, $\Delta H_{i}$. Defined in the three body axes as the difference between the components of the geomagnetic field, $\boldsymbol{H}$, and the one measured by the magnetometer. The maximum offset errors considered in the simulations are $\Delta H_{x}=1800 \mathrm{nT}, \Delta H_{y}=2010 \mathrm{nT}$, and $\Delta H_{z}=1400 \mathrm{nT}$. These offset errors are related to array $\boldsymbol{b}$ from Eqs. (4) and (6).

(3) Scale error in the three body axes, $k_{i}$. Defined in the three body axes as the ratio of the measured geomagnetic field to the geomagnetic field, $k_{i}=\boldsymbol{H}_{\boldsymbol{m} i} / \boldsymbol{H}_{\boldsymbol{i}}$. The maximum scale 

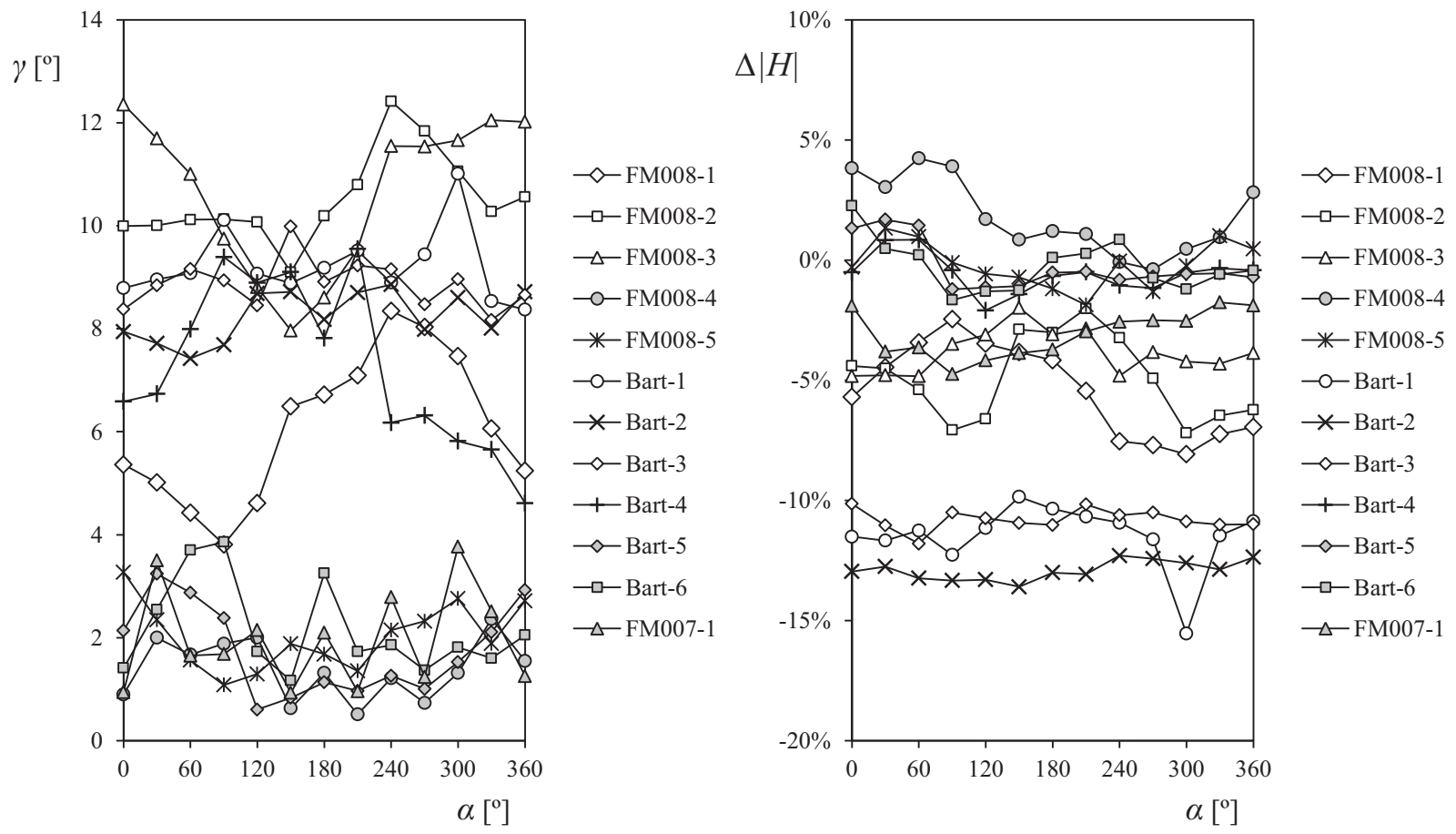

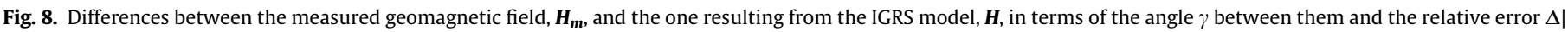
$\boldsymbol{H} \mid$, defined through Eq. (12), in relation to the angular position of the magnetometer, or its measurement probe, , within the testing procedure.

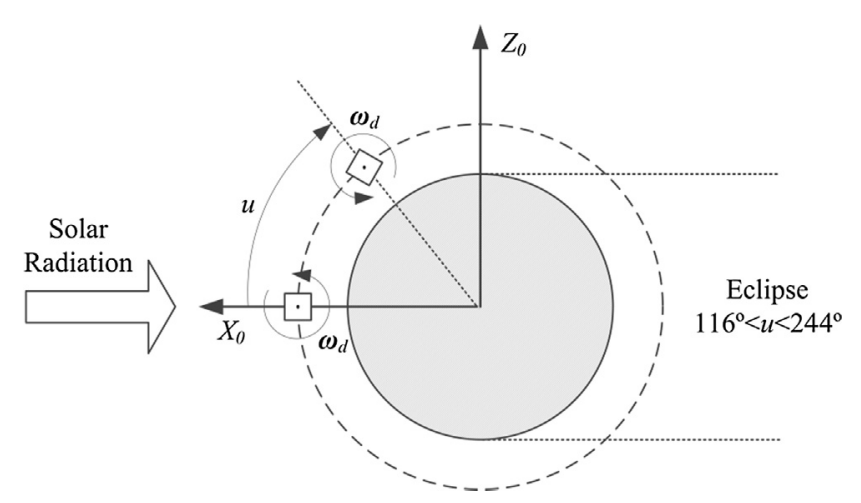

Fig. 9. UPMSat-2 satellite nominal attitude. The satellite is spinning around its $+Z$ axis, which is perpendicular to the orbit's plane, this orbit being LEO sunsynchronous (noon). From [31]. errors considered in the simulations are $k_{x}=1.1, k_{y}=1.2$, and $k_{z}=1.3$. The scale errors are mainly due to the scale factor from Eq. (7), with some contribution from the soft iron environmental error (Eq. (5)).

These errors have been introduced in Eq. (11) taking into consideration the following observations:

i. In matrix $C^{*-1}$ from Eq. (11), the diagonal elements are, at least, one order of magnitude bigger than the rest of the elements.

ii. The misalignment error that should be introduced in the non-diagonal elements is small $\left(2^{\circ}\right)$.

Therefore, we have considered that not taking into account the misalignment errors is acceptable. Thus, we are only introducing the scale and offset errors in the AOCS UPMSat2 simulator in Eq. (11), resulting in the three linear Eqs. (13a)-(13c).

$$
\begin{aligned}
& H_{m_{x}}=c_{x x}^{*} \cdot k_{x} \cdot b_{x}^{*}+c_{x y}^{*} \cdot b_{y}^{*}+c_{x z}^{*} \cdot b_{z}^{*}+\Delta H_{x} \\
& H_{m_{y}}=c_{x y}^{*} \cdot b_{x}^{*}+c_{y y}^{*} \cdot k_{y} \cdot b_{y}^{*}+c_{y z}^{*} \cdot b_{z}^{*}+\Delta H_{y} \\
& H_{m_{y}}=c_{x z}^{*} \cdot b_{x}^{*}+c_{y z}^{*} \cdot b_{y}^{*}+c_{z z}^{*} \cdot k_{z} \cdot b_{z}^{*}+\Delta H_{z}
\end{aligned}
$$

\subsection{Simulating the UPMSat-2 attitude control process}

The UPMSat-2 control law is a modification of the "B-dot" strategy that provides the control subsystem of the satellite with the capacity to control the rotation rate, and places the direction of the rotation axis perpendicular to the orbit's plane. This control law only needs magnetometers and magnetorquers, as sensors and actuators respectively, to carry out a successful attitude control on the spacecraft. The correct performances and applicability of the UPMSat-2 control law have been demonstrated by studying it with the UPMSat-2 mission simulator designed and programmed in MATLAB-SIMULINK by the IDR/UPM Institute. It is described in detail in [48]. The simulator integrates different modules which include:

- Inertia matrix of UPMSat-2.

- Accurate simulation of the expected orbit for UPMSat-2.

- A detailed geomagnetic field model based on NOAA 2015-2020 WMM.

- Models of the major perturbations along the orbit (e.g., torques due to solar and aerodynamic effects).

- Alternated operation of magnetometers and magnetorquers, to avoid interaction between them.

- A model of magnetorquers performance, which includes the response characteristic time and maximum magnetic torque.

- A model of magnetometers performance, with and without the errors aforementioned.

Three different stages have been observed within the control process: an initial correction of the rotation rate, followed by a cor- 
rection of the satellite's principal axis alignment, the process ending with a final stabilized stage. See in Fig. 10 a simulation of the UPMSat-2 ACDS performances starting from a perturbed initial state. In a first stage of the attitude control, the rotation speed components in the body frame $\omega_{x}$ and $\omega_{y}$ tend to zero, and $\omega_{z}$ approaches the target value $\left(\omega_{Z^{*}}=0.1 \mathrm{rad} / \mathrm{s}\right)$, the angle $\theta$ between the normal to the orbit and the satellite's $+Z$ axis being not controlled. This first stage ends in $t=t_{1}$ seconds after the beginning of the simulation (see Fig. 10). In the second stage, the rotation rate has been stabilized and the angle $\theta$ gradually tends to zero. This second stage ends in $t=t_{2}$ seconds after the beginning of the simulation (see Fig. 10).

A first round of simulations were carried out without considering any error on the magnetometers, in order to establish proper conditions to define the end of the first and the second staged of the stabilization (that is, times $t_{1}$ and $t_{2}$ ). The end of the first stage was established when the component of the satellite's rotation rate perpendicular to $+Z$ axis, $\omega_{\perp}$, is below $0.01 \mathrm{rad} / \mathrm{s}$, that is:

$\omega_{\perp}=\sqrt{\left(\omega_{x}\right)^{2}+\left(\omega_{y}\right)^{2}} \leqslant 10^{-2} \mathrm{rad} / \mathrm{s}$
As it can be observed, component $\omega_{z}$ is not included in the above condition, as this component of the rotation rate may not converge to the target value due to the magnetometer errors in the first stage. Despite this possible lack of convergence, the control law would be valid; because the ultimate goal of the control law is to minimize the angle, $\theta$, between the normal to the orbit and the direction of the angular momentum, as well as control the rotation rate as to enhance the thermal uniformity. As long as these goals are achieved, no matter if the value of $\omega_{Z}$ is slightly above or below the target value. Regarding to the condition related to the end of the second stage, it was set when the angle $\theta$ is below $10^{\circ}$.

\subsection{Results of the Monte Carlo analysis}

A Monte Carlo analysis consists of running about thousands of cases, one thousand cases can be considered a significant population, in a simulation platform, changing randomly the conditions of the simulation, as to obtain a statistical approach of the behaviour of the system simulated versus different conditions. In the
473

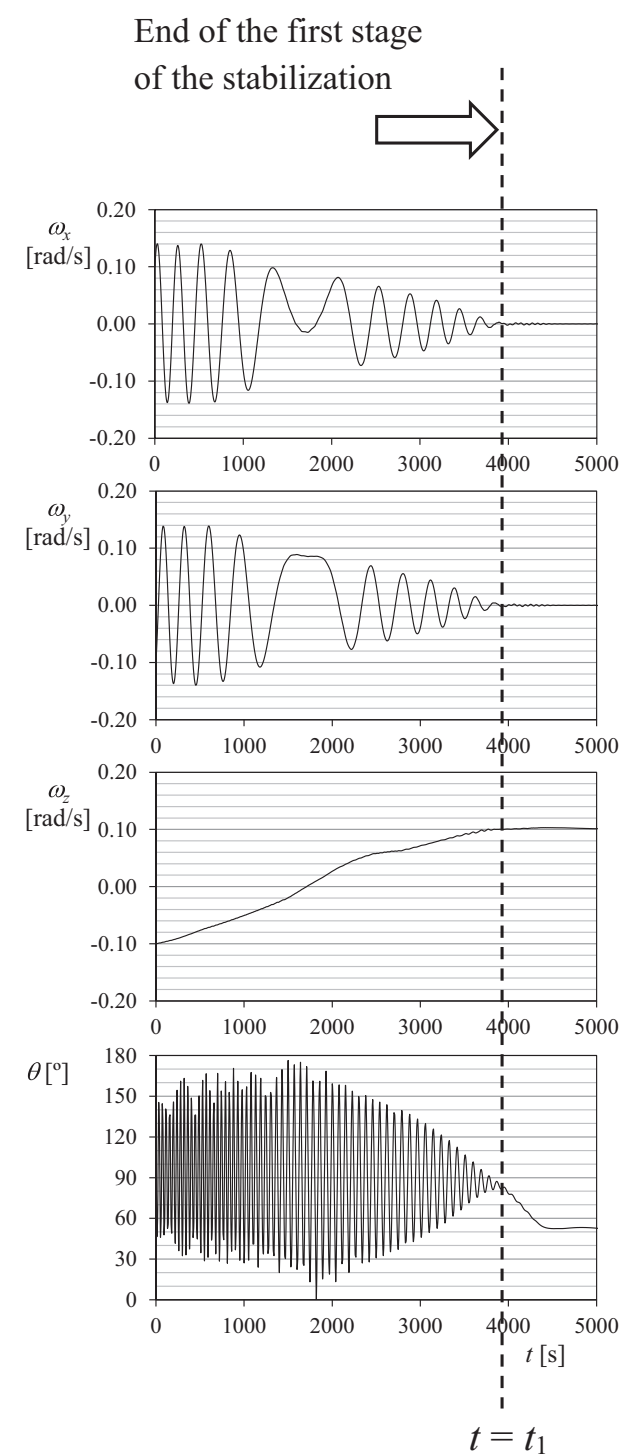

End of the second stage
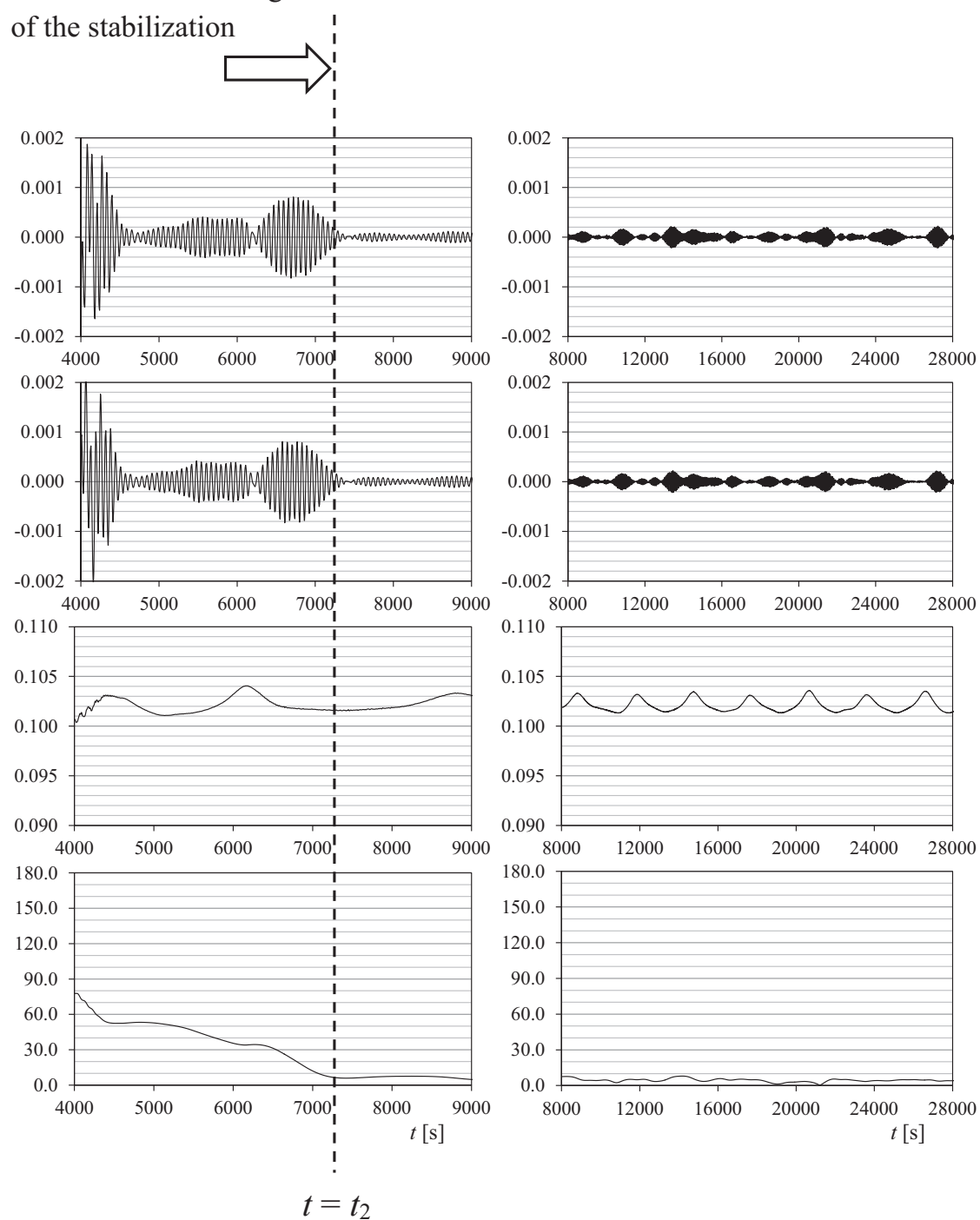

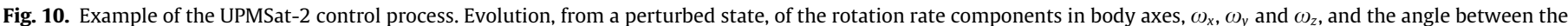

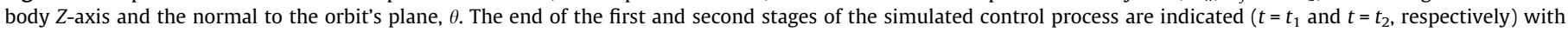
dashed lines. 

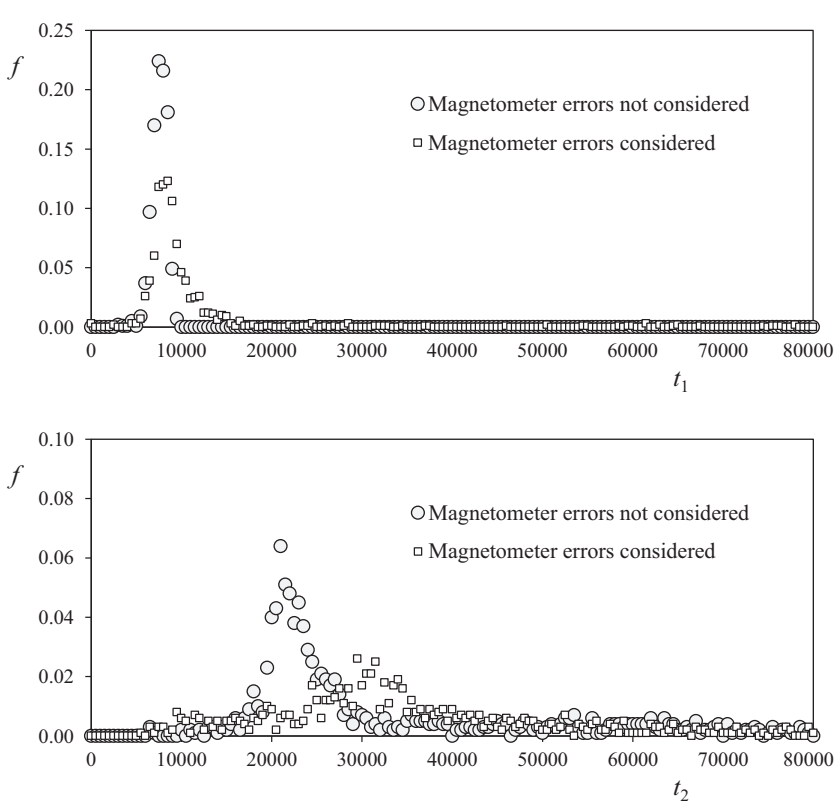

Fig. 11. Frequency histograms of times $t_{1}$ (top) and $t_{2}$ (bottom) corresponding to the 1000-case Monte Carlo simulations of the UPMSat-2 ACDS carried out considering and not considering the magnetometer errors.

Table 3

Times $t_{1}$ and $t_{2}$ in relation to different confidence levels from the distributions shown in Fig. 11.

\begin{tabular}{|c|c|c|c|c|}
\hline \multirow{2}{*}{$\begin{array}{l}\text { Confidence } \\
\text { levels }\end{array}$} & \multicolumn{2}{|l|}{$t_{1}[\mathrm{~s}]$} & \multicolumn{2}{|l|}{$t_{2}[\mathrm{~s}]$} \\
\hline & $\begin{array}{l}\text { No errors } \\
\text { considered }\end{array}$ & $\begin{array}{l}\text { Errors } \\
\text { considered }\end{array}$ & $\begin{array}{l}\text { No errors } \\
\text { considered }\end{array}$ & $\begin{array}{l}\text { Errors } \\
\text { considered }\end{array}$ \\
\hline $60 \%$ & 7623 & 8953 & 26,441 & 41,000 \\
\hline $70 \%$ & 7854 & 9717 & 34,250 & 5249 \\
\hline $80 \%$ & 8102 & 11,220 & 48,500 & 77,500 \\
\hline $90 \%$ & 8378 & 15,000 & 63,000 & 136,000 \\
\hline $95 \%$ & 8561 & 64,750 & 72,500 & 195,250 \\
\hline
\end{tabular}

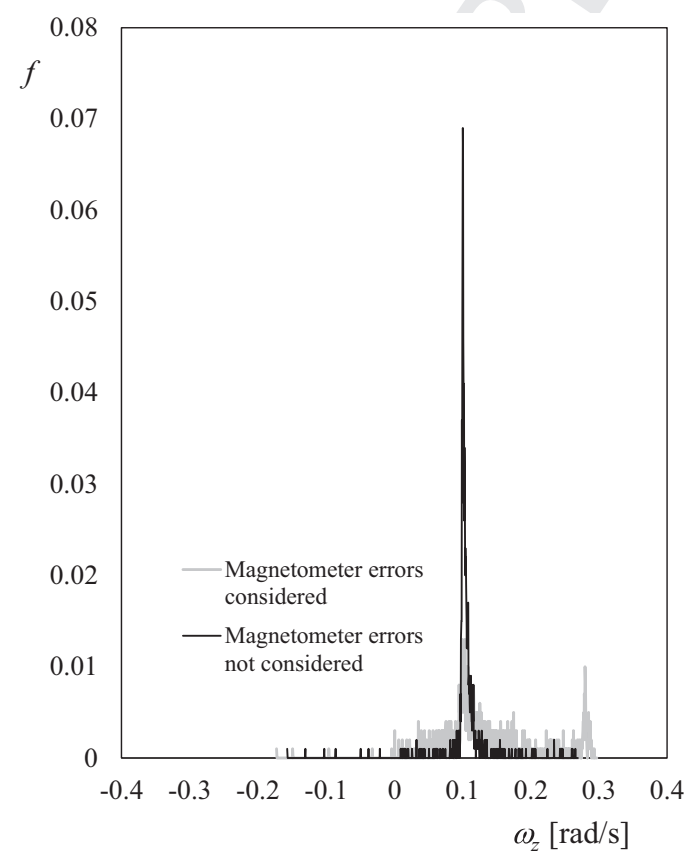

present work, two different simulations of one thousand calculated cases were ran, the following conditions being changed from one another calculation in each simulation:

- Initial angle between Greenwich longitude and the Aries direction.

- True Anomaly angle of the satellite.

- Initial quaternion (initial position of the body axes versus inertial axes-Earth fixed, $x$ pointing Aries Point, $z$ pointing earth rotation direction-).

- Initial angular velocity (in body axes).

All calculations were carried out for a period of $5 \cdot 10^{5} \mathrm{~s}$, which the maximum period of time that all the simulations will take.

Regarding the simulations, no magnetometer errors were considered within the first simulation, whereas misalignment, offset, and scale errors were taking into account within the second simulation. The effect of the magnetometer errors was analysed based on the end of the first and second stages of the stabilization process, $t_{1}$ and $t_{2}$, and the value of angle $\theta$ at the end of the first stage.

In Fig. 11 the frequency distribution of times $t_{1}$ and $t_{2}$ with regard to the two Monte Carlo simulations carried out are shown. The values of these times corresponding to $60-95 \%$ confidence levels in each simulation are respectively included in Table 3. As expected, values of $t_{1}$ and $t_{2}$ are larger when magnetometer errors are considered, being from 1.2 to 2 times the values from the nonerrors-considered simulation for confidence levels up to $90 \%$ (higher confidence levels indicate much greater differences). The following conclusions can be derived from Fig. 11:

- The introduction of the errors in the simulation distorts the values of the variables $t_{1}$ and $t_{2}$, so that the variance of the data is bigger in the sample with errors than in the sample without errors.

- The value of $t_{1}$ change slightly between the simulation with errors and without errors.

- Regarding $t_{2}$, the mean value increases when the simulation includes the errors in the magnetometers; the control spends more time in achieving the desired attitude.

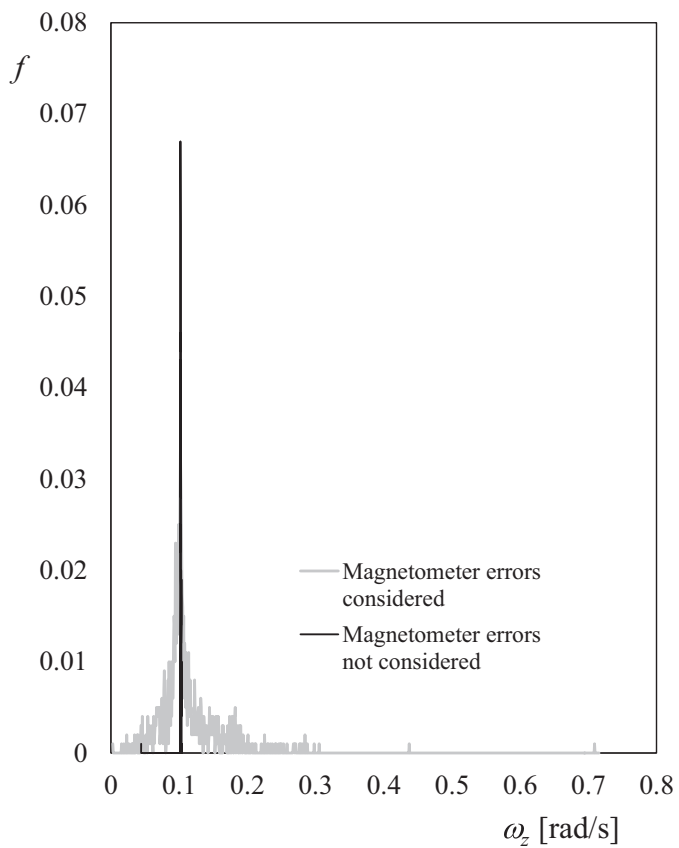

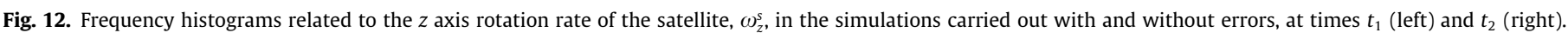


- There are fifteen cases that exceed the simulation time fixed $\left(5 \cdot 10^{5} \mathrm{~s}\right)$, because they do not reach the second condition established. We have ran these cases with a larger time of simulation $\left(10^{6} \mathrm{~s}\right)$, observing that most of them converge for times between $5 \cdot 10^{5} \mathrm{~s}$ and $10^{6} \mathrm{~s}$. It should be pointed out that the cases that exceed the time fixed represent only a $0.1 \%$ of the total number of cases analyzed, which is a negligible part of the whole sample.

In Fig. 12, the value of $\omega_{z}^{s}$ at times $t_{1}$ and $t_{2}$ is shown. As we have mentioned previously, the target value of $\omega_{z}^{s}$ is $0.1 \mathrm{rad} / \mathrm{s}$. As expected, the introduction of the errors in the simulation causes a bigger deviation from the mean value in $\omega_{z}^{s}$ at $t_{1}$ as well as at $t_{2}$; the values of the rotation velocity are more scattered in the cases with errors than in the cases without errors. In both simulations, with and without errors, most of the cases reach the target value of the variable, $0.1 \mathrm{rad} / \mathrm{s}$. However, in the simulation with errors we can observe cases that show at $t_{2}$ either bigger or smaller values than the target value. These cases correspond to those that do not converge for the time of simulation $\left(5 \cdot 10^{5} \mathrm{~s}\right)$. This larger period to reach the target value does not represent a problem; as long as the second condition $\left(\theta<10^{\circ}\right)$ is accomplished, that is, it does not matter if this component of the rotation velocity is slightly bigger or smaller than the target value.
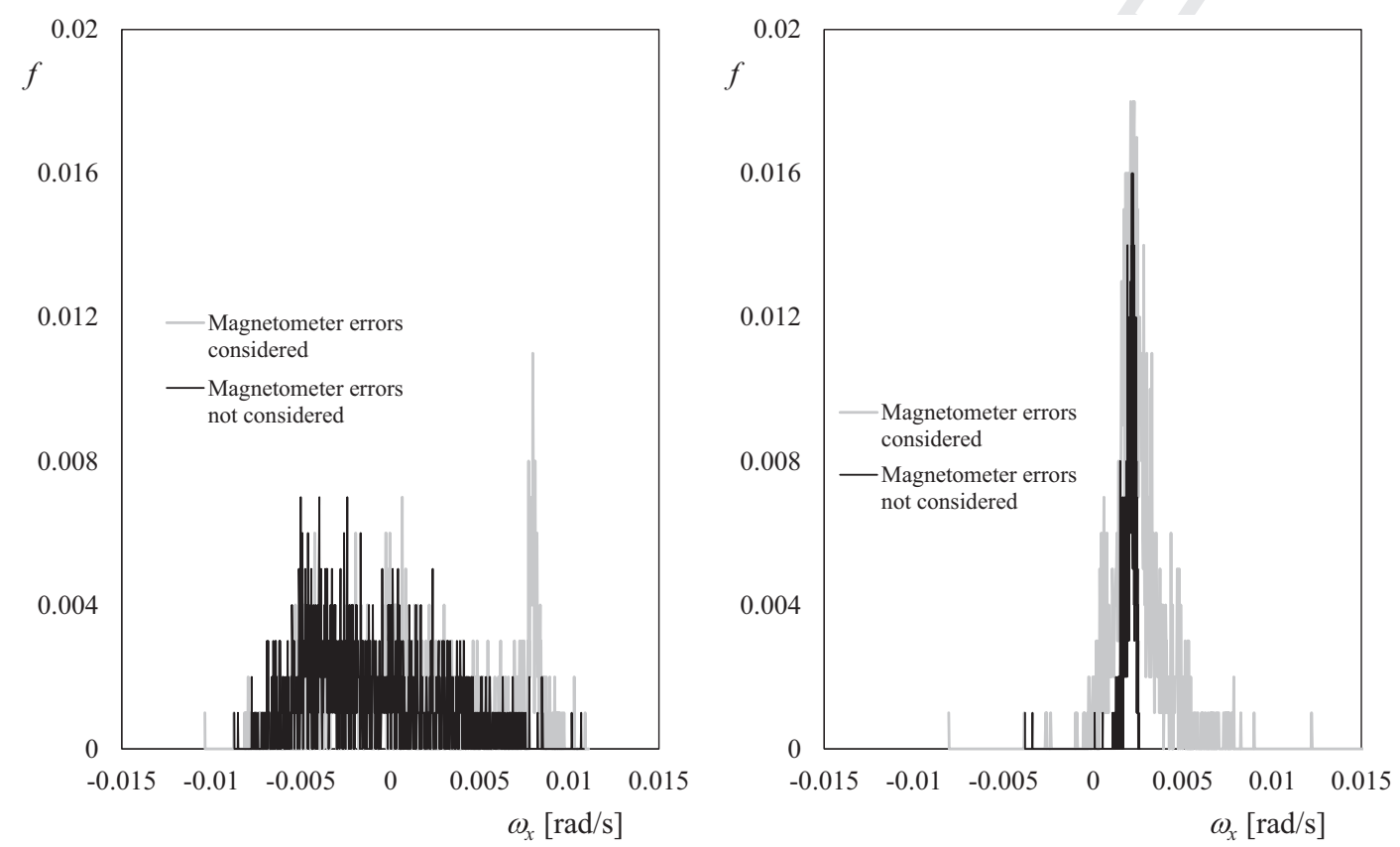

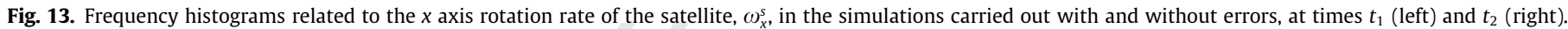
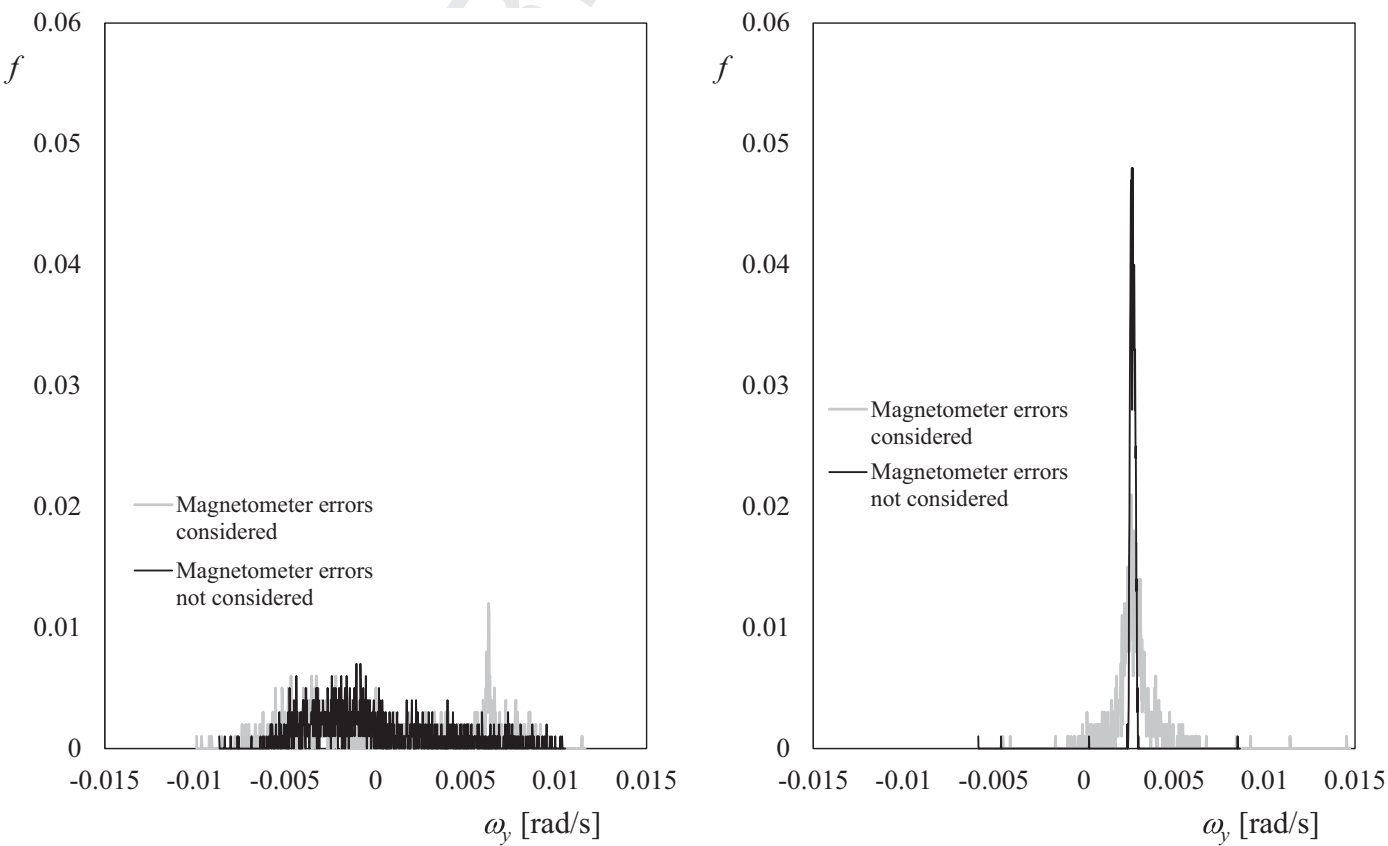

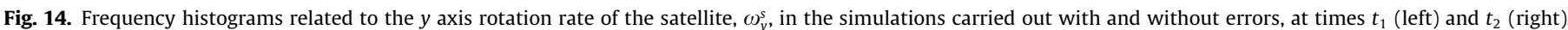


With regard the other two components of the rotation rate, $\omega_{x}^{s}$ and $\omega_{y}^{s}$, the frequency histograms of their values at times $t_{1}$ and $t_{2}$ is shown in Figs. 13 and 14. A quite bigger scatter is shown by both values at time $t_{1}$, resulting from the initial values programmed. Nevertheless, the situation is different at time $t_{2}$, where both components are around $0.002 \mathrm{rad} / \mathrm{s}$. As expected, the cases run without errors show a quicker convergence. Finally, $\omega_{y}^{s}$ rotation rate component seem to reach the convergence value easily when compared to $\omega_{x}^{s}$, this particular effect being produced by slight differences in relation to both components at the inertia matrix.

We can conclude that the control from the ADCS of the UPMSat2 is capable of overcoming the errors introduced in the magnetometer measurements, even though these errors have been oversized. Only less than a $0.1 \%$ of the cases have to be studied deeply because they have not converged in the time stipulated.

\section{Conclusions}

We have described the acceptance test over the ADCS and payload magnetometers (SSBV and Bartington respectively) from the UPMSat-2. The acceptance tests are recommended by the ECSS Test standard [32]. The purpose of these tests is to verify that the specifications of the elements represent their actual behaviour.

We have developed a test procedure simple and fast. It does not need specific equipment for its performance. The results of the test provide a first approach of the well-functioning of the sensors. For more exact results, especial support equipment to the test is needed. The most difficult requirement of the test to achieve has been an environment free from magnetic disturbances. We had to perform influence tests over all the support equipment, as to determine the influence of each element over the sensors. The suitable distances are established between the sensor and the support equipment during the test.

Regarding to the results, they allow us to determine the well behaviour of the sensors. As an example, to mention the results obtained in the tests performed indoors, shown in Fig. 8 for the Cases FM008-2 and Bartington Bart-1. As it can be seen, the vertical component of the Earth magnetic field measured presents a clear bias, which causes a bias in the modulus. This effect is supposed to be cause by the iron basement structure of the building. Thanks to the indoors' test, we could determine that it was inappropriate to do the magnetometer tests indoors.

As part of the acceptance test, we have been able of obtaining, using the tests results, a first definition of the errors that can be introduced by the sensors, magnetometers, in the ADCS. The goal has been to determine if the errors baseline could be assumed by the satellite AOCS. The definition of these errors have been very conservative, as to verify the good behaviour of the subsystem facing errors far bigger than the ones it is supposed to face. The errors have been coded in the programming language MATLAB/SIMULINK, inside a simulator of the ADCS previously designed and verified by colleges from the IDR Institute. A Monte Carlo analysis has been ran for samples of 1000 cases, for a simulation including the errors and a simulation without the errors. The consequences of these analyses have been shown using normal distributions of the most significant dynamic variables. The study of these graphs concludes that the control of the ADCS is capable of wellfunctioning even the oversized errors introduced in the magnetometer measurements. Although, there are few cases, less than $0.1 \%$, that do not show the expected behaviour; these cases should be studied more deeply in a future, even they can be considered negligible. Common characteristics observed in the variables corresponding to the simulation with errors are bigger variances in the values and longer times of convergence. Nevertheless, it is con- cluded that the results of this work are good enough as preliminary acceptance tests results.

\section{Acknowledgments}

The authors are indebted to the staff of the IDR/UPM Institute for their constant support. The authors are also grateful to the staff of the Library at Escuela Técnica Superior de Ingeniería Aeronáutica y del Espacio of Universidad Politécnica de Madrid, for their help in searching references and literature in relation to the magnetic control systems in space. Finally, the authors are grateful to Brian Elder for his help in improving the style of the text.

\section{References}

[1] J. White, F. Shigemoto, Satellite Attitude Control Utilizing the Earth's Magnetic Field, NASA TN, 1961

[2] A. de Ruiter, A fault-tolerant magnetic spin stabilizing controller for the JC2SatFF mission, Acta Astronaut. 68 (2011) 160-171, https://doi.org/10.1016/j. actaastro.2010.07.012.

[3] E.J. Findlay, A. De Ruiter, J.R. Forbes, H.H.T. Liu, C.J. Damaren, J. Lee, Magnetic attitude control of a flexible satellite, J. Guid. Contr. Dyn. 36 (2013) 1522-1527, https://doi.org/10.2514/1.57300.

[4] A.G. Cavallo, G. DeMaria, F. Ferrara, P. Nistri, A sliding manifold approach to satellite attitude control, in: Proc. 20th World Congr. IFAC, Sidney, 1993, pp. 177-184.

[5] L. Huang, W. Jing, Attitude stabilization of averaging magnetic torque, in: 1st Int. Symp. Syst. Control Aerosp. Astronaut., 2006, pp. 627-632, https://doi.org/ 10.1109/ISSCAA.2006.1627415.

[6] M. Chen, S.J. Zhang, F.H. Liu, Y.C. Zhang, Combined attitude control of small satellite using one flywheel and magnetic torquers, in: 2008 2nd Int. Symp. Syst. Control Aerosp. Astronaut., 2008, pp. 1-6, https://doi.org/10.1109/ ISSCAA.2008.4776367.

[7] R. Wisniewski, M. Blanke, Fully magnetic attitude control for spacecraft subject to gravity gradient, Automatica 35 (1999) 1201-1214.

[8] M. Lovera, A. Astolfi, Global magnetic attitude control of spacecraft in the presence of gravity gradient, IEEE Trans. Aerosp. Electron. Syst. 42 (2006) 796805, https://doi.org/10.1109/TAES.2006.248214.

[9] T. Bak, R. Wisniewski, M. Blanke, Autonomous Attitude Determination and Control System far the Orsted Satellite, 1996, pp. 173-186.

[10] R. Wisniewski, Satellite Attitude Control Using Only Electromagnetic Actuation, Aalborg University, 1996.

[11] P. Wang, Y.B. Shtessel, Y. Wang, Satellite attitude control using only magnetorquers, in: Syst. Theory, 1998. Proc. Thirtieth Southeast. Symp., IEEE, 1998, pp. 500-504.

[12] M.L. Psiaki, Magnetic torquer attitude control via asumptotic periodic linear quadratic regulation, AIAA 4043 (2000) 1-13.

[13] M. Lovera, A. Astolfi, Spacecraft attitude control using magnetic actuators, Automatica 40 (2004) 1405-1414.

[14] S. Barabash, I. Kiryushkin, O. Norberg, M. Ovchinnikov, V. Penkov, The nanosatellite MUNIN, a simple tool for auroral research, Adv. Sp. Res. 31 (2003) 313-318

[15] F. Santoni, M. Zelli, Passive magnetic attitude stabilization of the UNISAT-4 microsatellite, Acta Astronaut. 65 (2009) 792-803, https://doi.org/10.1016/j. actaastro.2009.03.012.

[16] M.Y. Ovchinnikov, A.A. Ilyin, N.V. Kupriynova, V.I. Penkov, A.S. Selivanov, Attitude dynamics of the first Russian nanosatellite TNS-0, Acta Astronaut. 61 (2007) 277-285, https://doi.org/10.1016/j.actaastro.2007.01.006.

[17] L. Stras, D.D. Kekez, G.J. Wells, T. Jeans, R.E. Zee, F. Pranajaya, D. Foisy, The design and operation of the canadian advanced nanospace eXperiment (CanX1), in: Proc. AMSAT-NA 21st Sp. Symp., Toronto, Canada, 2003, pp. 150-160.

[18] A. Aydinlioglu, M. Hammer, COMPASS-1 Pico Satellite: Magnetic Coils for Attitude Control, 2005, pp. 90-93.

[19] B. Graf, M.E.R.D. Gillet, SwissCube Control Algorithm Design and Validation, 2007

[20] S. Das, M. Sinha, K.D. Kumar, A. Misra, Reconfigurable magnetic attitude control of Earth-pointing satellites, Proc. Inst. Mech. Eng. Part G J. Aerosp. Eng. 224 (2010) 1309-1326, https://doi.org/10.1243/09544100JAERO681.

[21] S.P. Bhat, A.S. Dham, Spacecraft attitude under magnetic actuation, in: Proc. 42nd IEEE Conf. Decis. Control, Maui, Hawaii, 2003, pp. 2383-2388.

[22] S.S.P. Bhat, Controllability of nonlinear time-varying systems: applications to spacecraft attitude control using magnetic actuation, Autom. Control. IEEE Trans. 50 (2005) 1725-1735, https://doi.org/10.1109/TAC.2005.858686.

[23] R. Wisniewski, J. Stoustrup, Periodic H2 synthesis for spacecraft attitude contol with magnetorquers, J. Guid. Control. Dyn. 27 (2004) 874-881.

[24] M. Lovera, E. De Marchi, Periodic attitude control techniques for small satellites with magnetic actuators, IEEE Trans. Control Syst. Technol. 10 (2002) 90-95.

[25] J. Jung, N. Kuzuya, J. Alvarez, R.J. Twiggs, The design of the OPAL attitude control system, in: Proc. 10th Annu. AIAA/USU Conf. Small Satell., 1996, pp. 1-7. 
MEASUR 5848

No. of Pages 12, Model 5G

3 September 2018

12

E. Rodríguez-Rojo et al./Measurement $x x x$ (2018) $x x x-x x x$

690
691
692
693
694
695
696
697
698
699
700
701
702
703
704
705
706
707
708
709
710
711
712
713
714
715
716
717
718
719
720
721
722
723
724
725
726

[26] D. Rankin, D.D. Kekez, R.E. Zee, F.M. Pranajaya, D.G. Foisy, A.M. Beattie, The CanX-2 nanosatellite: expanding the science abilities of nanosatellites, Acta Astronaut. $\quad 57 \quad$ (2005) 167-174, https://doi.org/10.1016/j. actaastro.2005.03.032.

[27] J. Hales, M. Pedersen, K. Krogsgaart, Attitude Control and Determination System for DTUsat - A CubeSat Contribution, Technical University od Denmark, 2002.

[28] S. Pindado, E. Roibás-millán, J. Cubas, Á. Sanz, S. Franchini, I. Pérez-grande, G. Alonso, J. Pérez-álvarez, F. Sorribes-palmer, A. Fernández-lópez, M. Oguetagutiérrez, J. Zamorano, J. Antonio, D. Puente, A. Alonso, The UPMSat-2 satellite: an academic project within aerospace engineering education, 2nd Annu. Int Conf. Eng. Educ. Teach., 2017.

[29] S.P. Carlos de Manuel, Javier Cubas, On the simulation of the UPMSAT-2 microsatellite power, in: 10th Eur. Sp. Power Conf. Noordwijkerhout, 2001, pp. 751-757, https://doi.org/10.1115/1.1387022.

[30] J.A. De, P. Jorge, G. Emilio, J. Zamorano, A. Alonso, Using Internet-based Technologies in a University Satellite Project vol. 48 (2015) 82-86.

[31] J. Cubas, A. Farrahi, S. Pindado, Magnetic attitude control for satellites in polar or sun-synchronous orbits, J. Guid. Contr. Dyn. 38 (2015) 1947-1958, https:// doi.org/10.2514/1.G000751.

[32] ESA Requirements and Standards Division, Space engineering, ECSS-E-HB-10A, 2010.

[33] G. Sechi, G. André, D. Andreis, M. Saponara, Magnetic attitude control of the GOCE satellite, Eur. Sp. Agency, (Special Publ. ESA SP. 2005) (2006) 517-526.

[34] R.J. Patton, F.J. Uppal, S. Simani, B. Polle, Reliable fault diagnosis scheme for a spacecraft attitude control system, Proc. Inst. Mech. Eng. Part O J. Risk Reliab. 222 (2008) 139-152, https://doi.org/10.1243/1748006XJRR98.

[35] W. Wang, P. Menon, D. Bates, An Integrated Analytical/Numerical Framework for Verification and Validation of Attitude Control Systems for Flexible Satellites, ... Navig. Control .... (2012) 1-14. <http://arc.aiaa.org/doi/pdf/10. 2514/6.2012-4844>.

[36] P. Gasbarri, R. Monti, G. Campolo, C. Toglia, Control-oriented modelization of a satellite with large flexible appendages and use of worst-case analysis to verify robustness to model uncertainties of attitude control, Acta Astronaut. 81 (2012) 214-226, https://doi.org/10.1016/j.actaastro.2012.07.016.

[37] A. De Ruiter, Magnetic control of dual-spin and bias-momentum spacecraft, J. Guid. Control. Dyn. 35 (2012) 1158-1168, https://doi.org/10.2514/1.55869.
[38] G. Avanzini, E.L. de Angelis, F. Giulietti, Acquisition of a desired pure-spin condition for a magnetically actuated spacecraft, J. Guid. Contr. Dyn. 36 (2013) 1816-1821, https://doi.org/10.2514/1.59364.

[39] G. Avanzini, E.L. De Angelis, F. Giulietti, Spin-axis pointing of a magnetically actuated spacecraft, Acta Astronaut. 94 (2014) 493-501, https://doi.org/ 10.1016/j.actaastro.2012.10.035.

[40] P. Gasbarri, R. Monti, C. De Angelis, M. Sabatini, Effects of uncertainties and flexible dynamic contributions on the control of a spacecraft full-coupled model, Acta Astronaut. 94 (2014) 515-526, https://doi.org/10.1016/j. actaastro.2012.08.018.

[41] M. Lovera, Magnetic satellite detumbling: the b-dot algorithm revisited, in: Proc. Am. Control Conf. 2015-July, 2015, pp. 1867-1872, https://doi.org/ 10.1109/ACC.2015.7171005.

[42] V. Bohlouri, M. Ebrahimi, S.H.J. Naini, Robust optimization of satellite attitude control system with on-off thruster under uncertainty, in: 2017 Int. Conf. Mech. Syst. Control Eng. ICMSC 2017, 2017, pp. 328-332, https://doi.org/ 10.1109/ICMSC.2017.7959495.

[43] B.A.Z. Avoli, F.G. Iulietti, G.A. Vanzini, G.D.E. Matteis, Single Axis Pointing for Underactuated Spacecraft with a Residual Angular Momentum, 2017.

[44] B.G. Doerr, R. Linares, C.D. Petersen, Spacecraft attitude control using path integral method via Riemann manifold hamiltonian Monte Carlo, in: $2018 \mathrm{Sp}$. Flight Mech. Meet., 2018, pp. 1-15, https://doi.org/10.2514/6.2018-0204.

[45] Q. Hu, Y. Shi, X. Shao, Adaptive fault-tolerant attitude control for satellite reorientation under input saturation, Aerosp. Sci. Technol. 1 (2018) 1-12, https://doi.org/10.1016/j.ast.2018.04.015.

[46] H.S. Ousaloo, Attitude acquisition from an arbitrary tumbling state using two skewed reaction wheels, Aerosp. Sci. Technol. 72 (2018) 84-94, https://doi. org/10.1016/j.ast.2017.10.040.

[47] J. Jankowski, C. Sucksdorff, Guide for Magnetic Measurements and Observatory Practice, $1996 . \quad<$ http://iugg.org/IAGA/iaga_pages/pdf/IAGA-GuideObservatories.pdf>.

[48] L. Loubser, L. Newitt, Guide for Calibrating, 2009, pp. 1-35.

[49] N. Bowditch, The American Practical Navigator, Deffense Mapp. Agency Hydrogr. (2002) 882. <http://scholar.google.com/scholar?hl=en\&btnG= Search\&q=intitle:THE+AMERICAN+PRACTICAL+NAVIGATOR\#4>.

[50] U.S.D. of Comerce, National Centers For Enviromental Information, NOAA, 2016.
727

728

729

730
731

732

733

734

736

737

738

740

741

742

743

745

746

747
748

748
749

750

751

752

754

755

756

757
758

759

760

761
762

763

764

Please cite this article in press as: E. Rodríguez-Rojo et al., UPMSat-2 ACDS magnetic sensors test campaign, Measurement (2018), https://doi.org/10.1016/

j.measurement.2018.08.069 